\title{
Una nueva especie de Pentacalia (Senecioneae: Asteraceae) del Norte de Perú
}

\section{A new species of Pentacalia (Senecioneae: Asteraceae) from Northern Peru}

\section{Abundio Sagástegui y Eric F. Rodríguez}

Herbarium Truxillense (HUT), Universidad Nacional de Trujillo. Jr. San Martín 392. Trujillo, Perú. Email Abundio Sagástegui: abundiosag@hotmail.com Email Eric F. Rodríguez: efrr@unitru.edu.pe

\begin{abstract}
Resumen
Es descrita e ilustrada una nueva especie de Pentacalia Cassini (Senecioneae: Asteraceae) procedente del Departamento de La Libertad, Perú, y aparentemente endémica de la Provincia de Santiago de Chuco, denominada Pentacalia vallejiana Sagást. \& E. Rodr. sp. nov. Esta nueva especie es suigéneris entre las especies peruanas y dentro del género. Se compara con sus relacionados y adicionalmente se presentan datos sobre su distribución geográfica y ecológica.
\end{abstract}

Palabras clave: Pentacalia, Asteraceae, especie nueva, Santiago de Chuco, La Libertad, Perú.

\section{Abstract}

Pentacalia vallejiana Sagást. \& E. Rodr. sp. nov. is described as a new species of Pentacalia Cassini (Senecioneae: Asteraceae) from the Department of La Libertad, Peru. This new species is apparently endemic to the province of Santiago de Chuco. Pentacalia vallejiana is a remarkable Peruvian species and peculiar within the genus. It is compared with its closest relative and data on its geographical distribution and ecology are provided.

Keywords: Pentacalia, Asteraceae, new species, Santiago de Chuco, La Libertad, Perú.

\section{Introducción}

El género americano Pentacalia (Senecioneae: Asteraceae) fue descrito por Cassini en 1827 en alusión a la presencia de cinco costillas en los aquenios. Robinson y Cuatrecasas (1978) restablecieron el género olvidado por mas de 150 años, y en la actualidad presenta alrededor de 217 especies, distribuidas la mayoría en Sudamérica y en particular al norte de este continente (Díaz-Piedrahita y Cuatrecasas 1999).

Para la flora del Perú se han registrado alrededor de 50 especies, distribuidas en casi todos los hábitats, desde los bosques montanos, andinos, amazónicos, hasta las elevaciones altoandinas de la jalca y puna (Cuatrecasas 1981, 1994; Brako y Zarucchi 1993; Ulloa Ulloa et al. 2004), de ellas 32 son endémicas (Beltrán et al. 2006). El Perú ocupa el segundo lugar de especiación y diversificación por el número elevado de especies (Díaz-Piedrahita y Cuatrecasas 1999). Los últimos trabajos en este género para el Perú son los efectuados por Robinson \& Cuatrecasas (1993), quienes describieron 9 especies nuevas y una nueva combinación para la especie cusqueña $P$. vargasiana (Cabrera) H. Rob. \& Cuatrec.; Cuatrecasas (1994) realizó las nuevas combinaciones $P$. miniaurita (Sagást. \& M.O. Dillon) Cuatrec. y P. pleniaurita (Cuatrec.) Cuatrec.; así mismo, Beltrán (1999) efectuó la nueva combinación P. petiolincrassata (Cabrera \& Zardini) Beltrán.

Revisando críticamente tanto el material de herbario como el de las últimas colecciones procedentes de los andes peruanos, en particular del Norte con énfasis en la Región La Libertad (Prov. Santiago de Chuco), se ha encontrado una nueva especie, que denominamos Pentacalia vallejiana Sagást. \& E. Rodr. sp. nov. perteneciente a Pentacalia subgenero Pentacalia sensu Cuatrecasas (1981); cuya descripción, ilustración y discusión es el objetivo de este trabajo.

\section{Material y métodos}

El estudio está basado en la revisión de material único y observaciones directas de hábito y hábitat en el campo, efectuadas en el año 2009 en la localidad de Huayatán en la Provincia de
Santiago de Chuco, La Libertad; a 3098 m de altitud. Las colecciones se realizaron de acuerdo con la metodología y técnicas convencionales de herborización. Adicionalmente al trabajo de campo se fijó y conservó material en líquido (alcohol etílico al $70 \%$ o AFA) para estudiar la estructura floral.

El material botánico del tipo fue depositado en los siguientes herbarios: F, HUT, MO, US y USM. Son presentadas, la descripción, discusión, delineación y mediciones de la especie (Fig. 1A-J). También se adicionan datos de su ecología y distribución geográfica en la zona de Amotape-Huancabamba de elevada riqueza endémica (Weigend 2002; 2004). Los acrónimos de los herbarios son citados según Holmgren et al. (1990).

\section{Taxonomía}

\section{Pentacalia vallejiana Sagást. \& E. Rodr. sp. nov.}

$$
\text { (Fig. } 1 \mathrm{~A}-\mathrm{J})
$$

Tipo: PERÚ. Dpto. La Libertad, Provincia Santiago de Chuco, Huayatán (Stgo. de Chuco), $3098 \mathrm{~m}$, 808'44,2"S-78¹1'22,9”W, 23 Mayo 2009, A. Sagástegui A., E. León \& M. Félix 17669 (Holótipo: HUT; Isótipos: F, HUT, MO, US, USM).

Frutex ramosus ca. 2 m altus. Ramis fuscescentibus, glabrescentibus, irrigulariter 4-gonis, longe sulcatis, fistulosis, ca. $10 \mathrm{~mm}$ crassis. Folia alterna, petiolata (petiolis glabris, purpurescentibus, 2,5-3,5 cm longis), ovato-lanceolata, basi hastata, apice acuto-acuminata, irregulariter dentato-mucronulata, 13-16,5 cm longa, 2,5-3,5 cm lata. Auricula caduca, semicircular, ca. $1 \mathrm{~cm}$. Capitulescentiae cymoso-corymbiformes laxe terminales. Capitula multa, radiata, pedicelata (pedicellis $0,5-1,0 \mathrm{~cm}$ longis). Involucrum breviter conicum, 6-7 mm altum por 4-5 mm crassum. Caliculum 5-6 bracteolatum, bracteolis linearis, subaequalis, 1,5-2 $\mathrm{mm}$ longis, 0,5 $\mathrm{mm}$ latis. Phyllaria 13, 2-seriata; externis 5, linearis, acuminatis, 5-6 $\mathrm{mm}$ longis, 0,5-1 mm latis; internis 8, oblongo-linearis, acuminatis, 5-6 mm longis, 1-1,2 mm latis. Flores marginales 8 , feminei, ligulae 


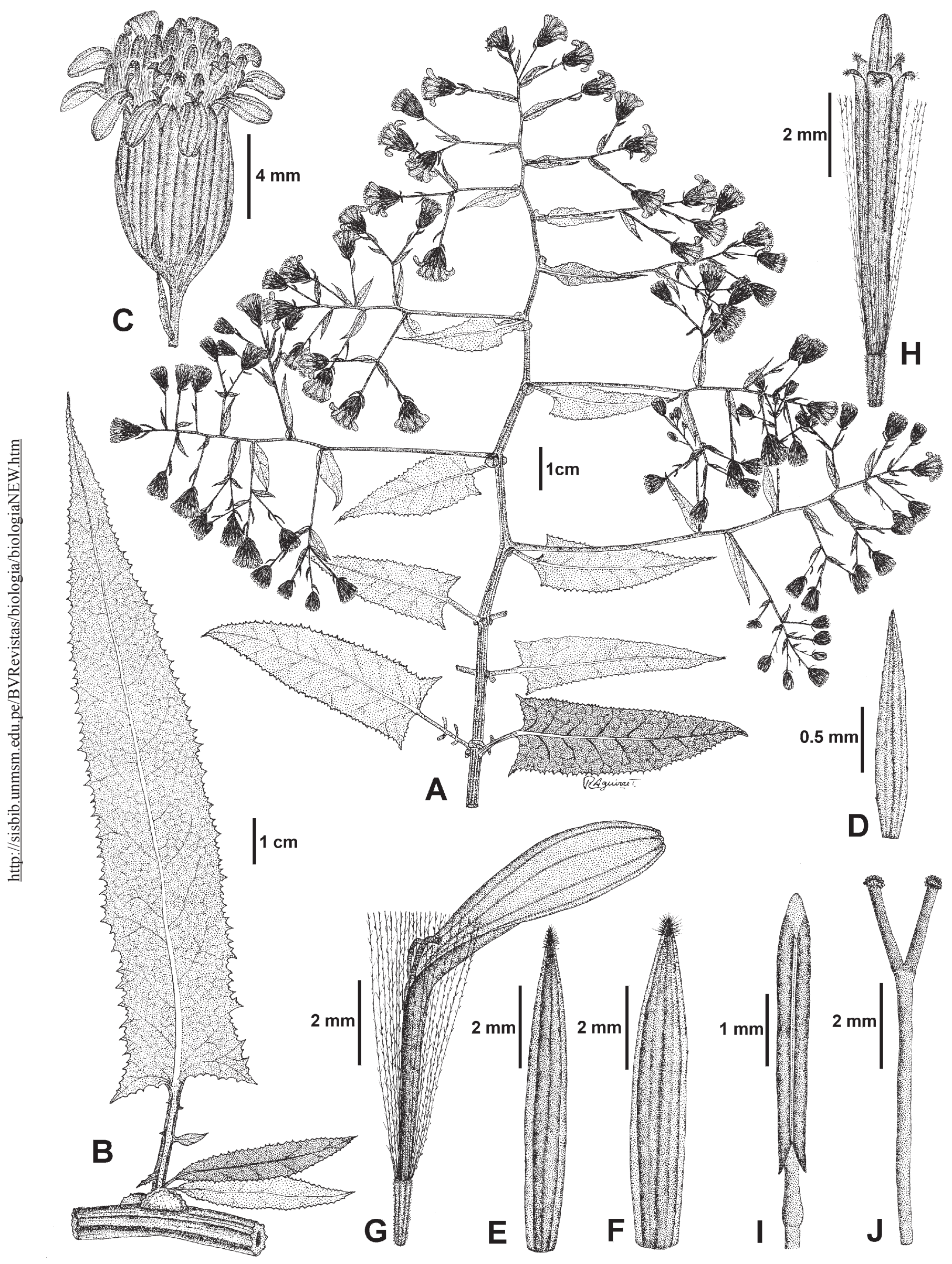

Figura 1. Pentacalia vallejiana Sagást. \& E. Rodr. sp. nov. A. Rama florífera; B. Hoja; C. Capítulo; D. Bracteola del calículo; E. Filaria externa; F. Filaria interna; G. Flor femenina ligulada; H. Flósculo; I. Antera; J. Ramas estigmáticas. Delineado por R. Aguirre T. de el holótipo A. Sagástegui A. et al. 17669 (HUT). 
oblongo-ellipticae, 4-nervatae, glabrae, 3-dentatae, 4-5 $\mathrm{mm}$ longae, 2-2,5 mm latae. Flores disci 26-28, hermaphroditi; corolla tubulosa, glabra, 7,5-8 mm, in tertio inferiore anguste, limbus quinquelobus. Achenia (immatura) oblanceolata, atrofuscescentes, glabra, 5-costata, 1,5-2,5 mm longa, $1 \mathrm{~mm}$ lata. Pappus uniseriatus, aristae 5-6 $\mathrm{mm}$ longae.

Arbusto apoyante, de unos $2 \mathrm{~m}$ de longitud. Tallos ramificados, irregularmente tetrágonos, surcados longitudinalmente, color marrón claro, glabrescentes, fistulosos, hasta $10 \mathrm{~mm}$ de diámetro. Hojas alternas, pecioladas (pecíolos ligeramente acanalados, purpúreos, glabros, 2,5-3,5 cm de largo), membranáceas; limbos desde ovados hasta ovado-lanceolados, hastados en la base, agudo-acuminados en el ápice, irregularmente dentadomucronulados, retinervados (nervadura central purpúrea), los mas grandes $13-16,5 \mathrm{~cm}$ de largo por 2,5-3,5 cm de ancho; aurículas 2, subcirculares, en la base del peciolo y rodean ligeramente al tallo, ca. $1 \mathrm{~cm}$ largo, caducas. Capitulescencias terminales en cimas corimbiformes laxas, blanco-tomentulosas (tomento caedizo), de hasta $17 \mathrm{~cm}$ de largo, bracteadas, brácteas angosto-lineares, 5-7 mm de largo. Capítulos numerosos, radiados, pedicelados (pedicelos delgados, marrones, de $0,5-1,0$ $\mathrm{cm}$ de longitud), 0,8-1,0 $\mathrm{cm}$ alto, $0,5-0,7 \mathrm{~cm}$ de diámetro. Involucro ligeramente cónico, verde pálido, de 6-7 $\mathrm{mm}$ de alto por 4-5 mm de diámetro. Calículo con 5-6 bracteolas lineares, subiguales, $1,5-2 \mathrm{~mm}$ de largo por $0,5 \mathrm{~mm}$ de ancho, dorso ligeramente blanco-lanuginoso. Filarias 13, 2-seriadas, 5 externas, lineares, cuspidadas y agudísimas, membranáceas, ligeramente engrosadas en el centro, márgenes tenuemente escariosos, porción apical penicelada, 5-6 $\mathrm{mm}$ de largo por $0,5-1 \mathrm{~mm}$ de ancho; 8 internas, oblongo-lineares, cuspidadas y agudísimas, engrosadas en el dorso, márgenes anchos y escariosos, porción apical penicilada, 5-6 $\mathrm{mm}$ de largo por $1-1,2 \mathrm{~mm}$ de ancho. Flores marginales 8 , femeninas, liguladas, $8-10 \mathrm{~mm}$ de largo y $2-2,5 \mathrm{~mm}$ en su parte mas ancha, tubo glabro de $4-5 \mathrm{~mm}$ de largo por $1 \mathrm{~mm}$ de ancho, lígula amarilla, 4-nervada, glabra, oblongo-elíptica, angostada y 3-dentada en el ápice, de 4-5 mm de largo por 2-2,5 $\mathrm{mm}$ de ancho; estilo exerto, 5-5,5 $\mathrm{mm}$ largo, ramas estigmáticas incurvadas, 1,5-2 mm largo; ovario oblongo, glabro, de 1-2 mm de largo papus blanco-piloso, setas $5 \mathrm{~mm}$ de largo, estrigosas. Flores del disco 26-28, hermafroditas, 7,5-8 $\mathrm{mm}$ de longitud, corola tubulosa, glabra, amarilla, estrecha en el tercio inferior, porción tubular 2,5-3 mm de largo por $1 \mathrm{~mm}$ de ancho, limbo 4,5-5 $\mathrm{mm}$ largo por 1,2-1,5 $\mathrm{mm}$ ancho, 5-lobulado, lóbulos triangulares de $0,7 \mathrm{~mm}$ de longitud por $0,5 \mathrm{~mm}$ de ancho, penicilados en el ápice; ovario oblongo, glabro, 1-2 mm de largo, papus blanco-piloso, setas $5 \mathrm{~mm}$ de largo, estrigosas; estilo brevemente exerto, 7,5-8,5 mm de largo, estigma bífido, ramas estigmáticas obtusas, truncadas, con corona de pelos dispersos en el ápice, 1,5 mm de largo; anteras exertas, 6,5-7,5 $\mathrm{mm}$ de longitud, tecas 3-3,5 $\mathrm{mm}$ de largo por $1 \mathrm{~mm}$ de ancho, apéndice apical oblongo $0,6 \mathrm{~mm}$ de largo, agudo-sagitadas en la base, caudas de $0,5 \mathrm{~mm}$ de largo, cuello lageniforme engrosado hacia la base, $0,5-0,7 \mathrm{~mm}$, porción libre del filamento 3-3,5 $\mathrm{mm}$ de largo. Aquenios inmaduros, oblanceolados, castaños (atroparduscos), glabros, de 1,5-2,5 mm de longitud por $1 \mathrm{~mm}$ de ancho, 5-costados; papus uniseriado, blanco-amarillento, cerdas de 5-6 $\mathrm{mm}$ de longitud.

\section{Discusión taxonómica}

Pentacalia vallejiana es una especie escandente, sólo conocida de la colección del tipo. Pertenece a Pentacalia subgénero Pentacalia sensu Cuatrecasas (1981), y suigéneris entre las especies peruanas y del género en general. Presenta calículos coniformes, hojas hastadas en la base, dos aurículas subcirculares caducas en la base del peciolo, combinación de caracteres no presentes en otras especies. Las especies con la presencia de aurículase se encuentran en Colombia (e.g.: P. genuflexa (Greenm.) Cuatr., P. rugosa (Cuatr.) Cuatr., P. favillosa (Cuatr.) Cuatr.); sin embargo difieren notablemente de la nueva especie.

\section{Distribución, ecología y fenología}

Se conoce de la localidad de donde procede el tipo, aparentemente endémica de esta región, creciendo a $3098 \mathrm{~m}$ de altitud en suelos negros de humificación variable; perteneciente a la denominada zona de Amotape-Huancabamba de elevada riqueza endémica y en su límite sureño (Weigend 2004). Especie apoyante asociada con otras Asteraceae y Agave americana, con quienes vegetan, generalmente a lo largo de los cercos de las chacras de cultivos.

Florece y fructifica entre mayo y julio después de las lluvias. Es visitado por abejas y otros insectos (planta melífera).

\section{Etimología}

Los autores tienen el honor y a la vez el placer y privilegio de dedicar este hallazgo al Dr. César Abraham Vallejo Mendoza (1892-1938), asociando de este modo al Vate Universal con una entidad florística igualmente universal y ambos nativos de la provincia Andina de Santiago de Chuco, Región La Libertad, Perú.

\section{Estado de Conservación}

Utilizando los criterios de la Lista Roja UICN (UICN 2001), esta especie endémica debería ser incluida en la categoría VU (Vulnerable), debido a que el área de presencia y ocupación es pequeña y sin protección por el Estado; así mismo, por el reducido número y tamaño de sus poblaciones que actualmente se encuentran afectadas por la ampliación de la frontera agrícola.

\section{Agradecimientos}

Agradecemos a los doctores Enrique León y Miguel Félix, de la Universidad Privada Norbert Wiener de Lima, Perú, por su colaboración en los trabajos de campo, y a la Bióloga Roxana Aguirre por la preparación de la excelente ilustración que forma parte de esta investigación.

\section{Literatura citada}

Beltrán, H. 1999. New combinanation in Dendrophorbium and Pentacalia (Senecioneae-Asteraceae) from Peru. Comp. Newsl. 34: 50-52.

Brako L. \& J. Zarucchi. 1993. Catálogo de las Angiospermas y Gimnospermas del Perú. Monogr. Syst. Bot. Missouri Bot. Garden. 45: 159.

Beltrán H.; A. Granda; B. León; A. Sagástegui; I. Sánchez \& M. Zapata. 2006. Asteraceae endémicas del Perú. En El libro rojo de las plantas endémicas del Perú. Ed.: B. León et al. Rev. peru. biol. Número especial 13(2): 130-134.

Cuatrecasas J. 1981. Studies on neotropical Senecioneae II. Transfers to genus Pentacalia of North Andean species. Phytologia 49(3): 241-260. 
Cuatrecasas J. 1994. Miscellaneous Notes on Neotropical Flora XXII. Identification of the genus Phyllostelidium and a few new combinations in the Compositae. Phytologia 76(5): 402-405

Díaz-Piedrahita S. \& J. Cuatrecasas. 1999. Asteraceae de la Flora de Colombia. Senecioneae-I, Géneros Dendrophorbium y Pentacalia. Academia Colombiana de Ciencias Exactas, Físicas y Naturales. Colección Jorge Álvarez Lleras $\mathrm{N}^{\circ} 12$.

Holmgren P. K.; N. H. Holmgren \& L. C. Barnett. 1990. Index Herbariorum. Part. I: The Herbaria of the World. $8^{\text {th }}$. ed. The New York Botanical Garden, Bronx, New York. U.S.A.

Robinson H. \& J. Cuatrecasas. 1978. A review of the Central American species of Pentacalia (Asteraceae, Senecioneae). Phytologia 40(1): 37-50.

Robinson H. \& J. Cuatrecasas. 1993. New species of Pentacalia (Senecioneae-Asteraceae) from Ecuador, Perú and Bolivia. Novon 3(3): 284-301.
UICN. 2001. Categorías y Criterios de la Lista Roja de la UICN. Versión 3.1. Preparado por la Comisión de Supervivencia de Especies de la UICN. UICN, Gland, Suiza y Cambridge, Reino Unido.

Ulloa Ulloa C.; J. L. Zarucchi \& B. León. 2004. Diez años de adiciones a la Flora del Perú: 1993-2003. Arnaldoa (Edic. Espec., Nov. 2004): 37-38.

Weigend M. 2002. Observations on the Biogeography of the Amotape-Huancabamba Zone in Northern Peru. In: K. Young et al., Plant Evolution and Endemism in Andean South America. Bot. Review 68(1): 38-54.

Weigend M. 2004. Additional observations on the biogeography of the Amotape-Huancabamba zone in Northern Peru: Defining the South-Eastern limits. Rev. peru. biol. 11(2): 127-134. 